UDC 811.112.2:811.111'373.45

DOI: $10.17223 / 24109266 / 10 / 12$

\title{
KRITISCHE ÜBERLEGUNGEN ZUR DEUTSCHEN SPRACHENPOLITIK DER GEGENWART IN RUSSLAND
}

\author{
Ju. Kobenko
}

\begin{abstract}
Resümee. Im Beitrag werden Voraussetzungen für eine ausgewogene deutsche Sprachpolitik in Russland im Fokus der gegenwärtigen Sprachsituation in der BRD erörtert. Im Mittelpunkt der Ausführungen stehen die Antinomie „Exo- vs. Endoglossie“ sowie der einschlägige begriffliche Apparat als Synthese solcher der Sprachkontaktforschung und Soziolinguistik. Das Hauptaugenmerk liegt hierbei auf dem Ausgleich sprachpolitischer Präferenzen, der bei der deutschen Sprachpolitikgestaltung im Hinblick auf Russland stattgegeben werden soll. Ein wesentlicher Teil des Beitrags ist der Kritik des bestehenden Sprachpolitikkonzepts gewidmet, welches sich durch eine übermäßig demokratische Auffassung der sprachpolitischen Variablen kennzeichnet. Die Analyseergebnisse der gegenwärtigen Sprachsituation in Russland lassen angesichts eines drastischen Statusschwundes des Deutschen ein stärkeres sprachkritisches Engagement der Öffentlichkeit sowie russischer Germanisten und eine unausbleibliche Revidierung des sprachpolitischen Gegenwartskonzeptes erwarten.
\end{abstract}

Schlüsselwörter: deutsche Sprachpolitik; Sprachkritik; Amerikanisierung; sprachpolitische Präferenzen; Sprachpolitikmodelle.

\section{Der Rückgang des Deutschen in Russland: Hintergründe und Auswirkungen}

Der dramatische Rückgang der deutschen Sprache in Osteuropa, vor allem in Russland, wo sie zumindest in der ersten Hälfte des XX. Jhd. fast die einzige Fremdsprache, das Mittel der Wissenschaftskommunikation und Völkerverständigung gewesen war, lässt sich im Allgemeinen auf eine Tatsache zurückführen: die übermäßig demokratische Auffassung der deutschen Sprachenpolitik von den Zeitgenossen in der BRD infolge einer weitgehenden Amerikanisierung der deutschen Sprache und Kultur. Die Symptome dieser Erscheinung allein im russischen Bildungswesen sind genau so gravierend wie unübersehbar und reichen von der Kürzung der Deutschstunden an russischen Hoch-, Mittelschulen und selbst an Gymnasien mit erweitertem Deutschunterricht über die Abschaffung des Deutschen als Fremdsprache überhaupt (z.B. an der Polytechnischen Universität in Tomsk, einer Stadt mit jahrhundertealter Deutschtradition), bis hin zur massenhaften - freiwilligen sowie erzwungenen - Konvertierung von Schulkindern und Studierenden an Hoch- und Berufsschulen zur Anglophonie. Einer repräsentativen Umfrage aus dem Jahr 2011 zufolge lernte nur jeder Zehnte an den insgesamt sieben Tomsker Universitäten Deutsch, jeder Fünfzehnte - Französisch. 
Im heutigen Russland wird Deutsch vorwiegend von denjenigen - ob Germanisten oder nicht - gelernt, die in Deutschland bessere Lebensverhältnisse sehen und dort aus diesem Grund einen Ehepartner suchen; dazu steht Ihnen das großzügige Kommerzangebot der russischen Vertretungen des Goethe-Instituts zur Verfügung. In äußerst seltenen Fällen wird die Erlernung dieser Sprache durch kulturhistorisches Interesse motiviert, vielmehr gilt das Interesse aber dem hohen Lebensstandard und guten Erwerbsperspektiven. Diese beinahe erniedrigende Ausstattung des gegenwärtigen Funktionsrahmens der Sprache von Klopstock, Brecht und Grass ist ein Produkt der zügellosen Demokratisierung und Verwirtschaftlichung der deutschen Kultur seit dem Kriegsende. Die akademische Tradition der russischen Germanistik, die ehemals solche großtönende Namen wie Žirmunskij, Moskal'skaja, Guchman, Semenjuk, Domaschnev, Devkin usw. kannte, verkümmert zusehends und lässt um die Germanistikfakultäten nicht nur im Transuralgebiet, sondern auch in Zentralrussland fürchten. Jüngere Köpfe, die sich eine krisenfeste Zukunft sichern möchten, haben längst auf Englisch umgesattelt und wollen nichts mit den ,aussterbenden Sprachen Europas“ zu tun haben, zumal sie bereits Erfahrung gemacht haben, dass deutsche Staatsbürger im Ausland auch nur Englisch reden. Deutsche Germanisten berichten über Deutsch in ihrer Heimat noch weniger Erfreuliches: „Vor kurzem tagte in Berlin eine Konferenz mit dem schönen Titel „Gedankenforscher“. Alle Referenten - sechs Deutsche, drei aus den Vereinigten Staaten, ein Brite - waren hervorragend. Und alle sprachen Englisch oder, im Fall der deutschen Redner, mitunter auch so etwas Ähnliches. Seltsam gewählte Worte und verschlungene Sätze ließen so manchen Vortrag weniger brillant wirken, als er inhaltlich war. Wer aber sprach im Publikum Englisch? Niemand. Und auch die vier ausländischen Redner hätten einen deutschen Vortrag ohne Mühe verstanden, denn überall lagen Kopfhörer für die Simultanübersetzung bereit. Selbstverständlich habe man es sich gewünscht, dass die einheimischen Redner Deutsch sprächen, erklärte mir die finanzierende Stiftung. So wäre die Resonanz in der Öffentlichkeit stärker gewesen. Aber die Professoren wollten es anders. Ihr Argument: Nur eine Veranstaltung mit Konferenzsprache Englisch nehme man ernst" [3].

„Deutsch entwickelt sich in Deutschland zunehmend zu einem Freizeitdialekt", prophezeit Günther Öttinger in Harold Woetzels spektakulärem Film „Wer rettet die deutsche Sprache?“ (2007), der für viele im Ausland ein Hoffnungsträger war. In Russland sowie fast überall im postsowjetischen Raum gewinnt Deutsch langsam das Attribut einer exotischen Sprache, die nur das onomasiologische Sachfeld „Deutschtum“ bedient. Für alles andere in der Welt scheint es schlicht Englisch zu geben. Ein Jahrhundert deutscher Geschichte mit seinen zahlreichen Basiserfindern und knapp 90 deutschsprachigen Nobelpreisträgern, fundamentalen Errungenschaften in Naturwissenschaften und Technik, Auszeichnungen in Lite- 
ratur und Friedenspolitik fiel im Heimatland völlig überraschend dem Amerikafimmel zum Opfer [12: 940].

Die Amerikanisierung wird in diesem Beitrag zweierlei verstanden: im intralinguistischen Sinne als inflationär gewordener Gebrauch von AngloAmerikanismen bzw. diesbezügliches Sprechverhalten der Deutschen, die z.B. das französische Wort „Journal“ nicht mehr auf französische Weise, sondern mit einer englischsprachigen Affrikate [dz] aussprechen; im extralinguistischen Sinne betrifft der Begriff die Vorstellungen vom Funktionieren der deutschen und anderen Sprachen in bestimmten Verbreitungsgebieten, ihre sprachenpolitischen Variablen, Statusparameter usw.; unter diesem Gesichtspunkt versteht sich die Amerikanisierung hier als eine bedingungslose Kapitulation der Deutschen vor den Folgen der aggressiven Verbreitung des Englischen weltweit und die Einräumung seiner Vorrangstellung im eigenen Land und zwangsläufig überall in Europa (sic!).

Die Mehrheit der in Russland tätigen Deutschen, darunter vor allem DAAD-Lektoren und Mitarbeiter des Goethe-Instituts, üben sich gern in „politischer Korrektheit“" gegenüber dem invasiven und erfolgreicheren Englischen und offerieren ihren russischen Fachkollegen ein Konzept der Sprachenpolitik mit Deutsch in der Rolle einer Zweitfremdsprache. Es ist jedoch unklar, auf welches Areal das Konzept zu beschränke wäre, denn sprach(en)politische bzw. sprachökologische Konzepte funktionieren in konkreten Rahmenbedingungen, aber von vornherein ist klar, in Russland ist diese Vision aus folgenden Gründen schlecht realisierbar: erstens funktioniert hier über knapp 17 Mio. Quadratkilometer eine einzige Staatssprache eine sprachpolitische Dimension, die in der Welt nicht ihresgleichen hat; bei dem Mindestma $ß$ an akzeptierter Sprachdispersion, einer geschichtlichen Bedingtheit, fällt es vielen Russen schwer, überhaupt eine erste Fremdsprache zu erlernen, ganz zu schweigen von einer zweiten, für die selbst diplomierte Philologen meistens keinen Gebrauch machen können; zweitens lautet die didaktische Weisheit für das Sprachenpaar Deutsch und Englisch wie folgt: zuerst Deutsch und dann Englisch, nicht umgekehrt, wenn man beide Sprachen passabel beherrschen möchte. Bei der „Englisch 1 - Deutsch 2“Kombination mehren sich in Zukunft statt hervorragender Germanisten lediglich Schwänzer und Fünfenschreiber und die Kenntnisse der zweiten Fremdsprache tendieren ein Jahr nach dem Abitur gegen null.

Leider lässt sich nicht feststellen, ob diese Vision von einem deutschen Sprachenpolitiksubjekt oktroyiert ist oder man hier eher mit einer Menge von übereinstimmenden laienhaften Sprachenpolitikauffassungen zu tun hat, die bloß Ableitungen von der gegenwärtigen Sprachsituation in der BRD sind. Noch weniger klar ist es, auf welchem Wege das Konzept im Vielvölkerland Russland mit zahlreichen hiesigen Kleinsprachen als Bildungskomponenten und anderen traditionell unterrichteten Fremdsprachen wie Französisch, das didaktisch gesehen zu Englisch besser passen würde, in 
die Praxis umgesetzt werden soll. Will man es einschließlich oder nur in Russland realisieren und soll die „English-first-Policy“, die erfolgreich in Deutschland umgesetzt wurde und als Folge der Amerikanisierung anzusehen ist, ein Beispiel für ganz (Ost)Europa werden?

Höchst polemisch erscheint auch die dahin gehende Argumentation einiger deutschsprachiger Germanisten, dass „man die Interessen der Lernenden in Russland in den Vordergrund stellen muss, bevor man die Werbetrommel für Deutsch rührt". Dennoch bekommt man von selbigen Kollegen keine Antworten auf die hieran anschließenden Fragen,

- wie es Deutsch dann überhaupt schaffen kann, gegen die aggressive und massiv unterstützte transkontinentale Verkehrssprache Englisch im größten Land der Erde anzukonkurrieren, wenn sich selbst in den Muttersprachlern kein Funke zur Konkurrenz entfachen lässt?

- wäre es nicht logisch, den Vorrang des Englischen in Frage zu stellen wenigstens in einer Situation, wo es heißt, um Lernende zu kämpfen?

- wie und inwiefern wäre es der deutschen Sprache in Russland selbst förderlich, wenn man die Meinungen von Kindern einholt, die in einer Welt aufwachsen, wo vieles Englisch (oder Halbenglisch) klingt?

- wäre die weltumspannende ,as-default"-Anglophonie nicht Beweis genug, dass die Interessen der Lernenden beim Fremdspracherwerb nicht im Vordergrund stehen bzw. sowieso nicht wahrgenommen werden?

- was, wenn die Interessen nur einem besseren Leben in Deutschland gelten; soll dafür die Werbetrommel gerührt werden?

Aber seltsamerweise hört man genau von der deutschen Seite, die in dieser Partie am stärksten benachteiligt sein sollte, kein Wort der Kritik hinsichtlich der Allgegenwart des Englischen. Die Haltung vieler deutscher Kollegen weckt den Eindruck, als hätten sie sich mit der Situation längst abgefunden und als ließe sich an der bestehenden Rangordnung der Fremdsprachen in der BRD sowie in Russland nichts mehr ändern. Eine produktive Sprachkritik, gewisse Paritätsvorschläge, Anregungen zu einer gesunden Konkurrenz der Fremdsprachen in Russland fehlen als solche. Stattdessen lässt sich eine fast billigende, unterwürfige Einstellung gegenüber dem Englischen erkennen.

Große Hoffnungen setzen die Deutschen hingegen in eine Neufassung der föderalen Bildungsstandards (rus. FGOS) des russischen Bildungsministeriums, wonach an allgemeinbildenden Schulen der Russischen Föderation bereits im Herbst 2016 ab 5. Klasse eine zweite Fremdsprache verbindlich wird. Ohne Zweifel hat Deutsch hier gute Chancen, dank seiner reichen Tradition in Russland in schulischen Lehrplänen $\mathrm{Fu} ß \mathrm{zu}$ fassen. Allerdings verbinden die Systemkritiker diese umstrittene Neuerung weniger mit dem Wunsch von Ex-Bildungsminister Livanov „Intellekt und Gedächtnis der Schüler zu fördern“, sondern lediglich mit dem proamerikanischen Charakter der Bildungsreformen im russischen Bildungswesen: „Die Einführung einer 
zweiten Fremdsprache führt dazu, dass die Schüler ihre Muttersprache komplett vergessen, dabei werden die Kenntnisse der anderen Fremdsprache sowieso mangelhaft sein" [11]. Bei der glücklichsten und vielerorts höchstwahrscheinlichen „Englisch 1 - Deutsch 2“-Kombination wird dem Deutschen eine a priori nachteilige Position beschieden, denn der Grundsatz der fundamentalen sowjetischen Theorie des Fremdsprachenlernens lautet: um die beiden Sprachen gleichermaßen gut zu beherrschen, benötigt Deutsch fast das Dreifache an Zeitaufwand als Englisch. Ginge es dem russischen Bildungsministerium wirklich um die Qualität, würden die FGOS mit einem entsprechenden Vermerk versehen. Für das Deutsche aber wäre auch diese Kombination ein großer Gewinn, so prekär ist dessen Status in Russland, welches früher neben den USA zu den bedeutendsten Zentren der Auslandsgermanistik weltweit zählte.

Aber das durchaus Schlimmste, das der deutschen Sprache in Russland wiederfahren kann, ist der Mangel an qualifizierten Lehrkräften. Der Beruf eines Deutschlehrers hat infolge des drastischen Rückgangs des Deutschen erheblich an Attraktivität eingebüßt. Als Folge werden landesweit Lehrstühle für Deutsch geschlossen bzw. mit denen für Französisch zusammengelegt; Fremdsprachenfakultäten, an denen größtenteils Englisch unterrichtet wird, werden philologischen Einrichtungen angeschlossen, der Kaderabbau eilt an pädagogischen Universitäten, die ehemals und vereinzelt heute noch die staatliche Plattform für die Berufsausbildung von Fremdsprachenlehrern waren, jährlich von Rekordhoch zu Rekordhoch. Die Ausbildung von Germanisten wird allmählich aus dem universitären Bereich in die meistens von NROs veranstalteten Kurse oder sogar nach Deutschland verdrängt. Angesichts solcher Veränderungen folgt die unausweichliche Frage nach der Nachfolge der reichen germanistischen Tradition in Russland: kommt es genauso wie bei den deutschen Sprachinseln im Ausland zum „Kontinuitätsbruch“ nach K.J. Mattheier [7: 724] oder werden im Sanktionskrieg beide Traditionen - die germanistische in Russland und die slawistische in Deutschland - zeitgleich untergehen?

\section{Sprachhistorische und soziolinguistische Merkmale der deutschen Sprachenpolitik: Ein systematischer Überblick}

Die deutsche Sprach- sowie Sprachenpolitik ist traditionell durch einen Polyzentrismus der Normengebung gekennzeichnet, der in seinen wesentlichen Zügen mindestens seit der Wiedervereinigung fortbesteht. Beispielsweise beschäftigen sich im heutigen Deutschland etwa 24 sprachpolitische Subjekte (19 Sprachvereine, 2 Sprachstiftungen und 3 staatliche geförderte Sprachinstitutionen) mit der Normengestaltung der deutschen Gegenwartssprache, wodurch die Normenvielfalt und -varianz erheblich gesteigert wird. Auch die Kodifizierungsmaßnahmen unterscheiden sich von Subjekt zu Subjekt derart massiv, dass man in Einzelfällen nahezu von gegensätzli- 
chen sprachpolitischen Konzepten (z.B. bei der Dudenredaktion und dem Verein deutsche Sprache) ausgehen könnte.

Die aktuelle Sprachenpolitik in der BRD hinsichtlich der zwei wichtigsten Komponenten der Sprachsituation im Land seit 1945 - des importierten englischen Metalekts und der deutschen Schriftsprache als Sprachsituationsexponent - verzeichnet einen akuten Mangel an Standardisierungsmaßnahmen angesichts der überbordenden Zahl von Anglo-Amerikanismen im deutschen Sprachgebrauch der Gegenwart. Im krassen Wiederspruch stehen einerseits die Beflissenheit der Massenmedien, die deutsche Sprache mit immer neuen englischsprachigen Fremdwörtern zu versetzen, und andererseits der geschichtlich bedingte Polyzentrismus der deutschen Sprach- und Sprachenpolitik sowie der damit zusammenhängende Einheitlichkeitsmangel an Sprachnormen, dieser Flut an Anglo-Amerikanismen effizient entgegenzuwirken. Die heutige Sprachnormenlandschaft in Deutschland befindet sich auf der Skala der sprachpolitischen Präferenzen unzweideutig links außen und entspricht hiermit dem exoglossischen Pol, zu dessen unterscheidenden Merkmalen Normenvielfalt, Konzept der sog. Sprachdemokratie, daraus resultierendes Vorzug der Interessen einer Sprachperson gegenüber den des Sprachkollektivs und Permissivität der Normengebung gezählt werden können.

Dem Allensbacher Bericht „Was denken die Deutschen über ihre Muttersprache und Fremdsprachen?" aus dem Jahr 2008 ist zu entnehmen, dass die widersprüchliche sprachenpolitische Situation zu einer Spaltung der Sprach- und somit Identitätsauffassung in Deutschland führen kann: die ältere Generation spricht sich ausdrücklich für die Verschärfung der Kodifizierungsmaßnahmen, wohingegen die jüngere sich eher für einen lockeren Umgang mit der Titelsprache einsetzt. Diese Interessendiskrepanz legt ein beredtes Zeugnis über den Einheitlichkeitsmangel der gegenwärtigen deutschen Sprach- und Sprachenpolitik $\mathrm{ab}$, der am besten durch ein ausgewogenes sprach(en)politisches Modell überwunden werden kann. Während die Exoglossie als sprach(en)politische Präferenz mit der Veränderung einer Sprache im Zusammenhang steht, steht der entgegengesetzte endoglossische Pol auf der Skala der sprachpolitischen Präferenzen für Spracherhaltung und -wahrung. In seiner Geschichte pendelte das Deutsche stets von einem Pol zum anderen: Momente der Strenge wurden unausweichlich durch ihre Demokratisierung abgelöst [9: 42].

$\mathrm{Zu}$ fragen wäre, wann wird die exoglossische Phase der heutigen Sprachenpolitik endlich durch die endoglossische abgelöst, von der die deutsche Sprache und die gesamte Germanistik profitieren soll? Was muss getan werden, damit die deutsche Sprachenpolitik bewusster, einheitlicher, zentralistischer und konsequenter wird?

In seinen „Maximen und Reflexionen“ erwähnt J.W. von Goethe ein Modell der Sprachnutzung, bei dem ,der affirmative Purismus als Grundlage einer besonnenen Entlehnung vorwalte". Der meistenfalls noch so negative empfundene Purismus hat nichts mit Restriktion und Sprachdeskriminierung 
zu tun, sondern vielmehr mit der Besinnung auf die Schöpfungskraft der eigenen Sprache. Das zentristische Funktionsprinzip des Deutschen, das hier angedeutet wurde, mündet in das Konzept eines exo-endoglossischen Sprach(en)politikmodells, das ein Ausgleich zwischen dem poly- und monozentristischen, sprachdemokratischen und -nationalistischen, permissiven und restriktiven Charakter der Sprachstandardisierung darstellt [5: 1020]. Eine Notlösung könnte eine deutsch-englische Diglossie in den am meisten mit englischem Sprachgut durchsetzten lexikalischen Bereichen des deutschen Sprachbestands sein, zu denen nach D. Herberg die der Computer- und Internettechnologien, der Massenkommunikation, des Gesellschaftslebens, des Sports und der Finanzen gehören [2: 198]. Das Konzept eines exoendoglossischen (zentristischen) sprach(en)politischen Modells setzt eine Korpus- und Statusplanung der Sprachsituationskomponenten voraus. Außerdem werden die korpus- und statusbezogenen Präferenzen laut W.T. Klokov auf Verwaltungs-, Wissenschafts- und Gesellschaftsebenen verwirklicht [4: 57]. In der Korpusperspektive könnte die polyzentristische Dynamik durch folgende Maßnahmen ausgeglichen werden: auf der Verwaltungsebene könnten die Kodifizierungsanforderungen für Anglo-Amerikanismen zur Verhinderung der redundanten Rechtschreibvarianz unifiziert werden; auf der Wissenschaftsebene wäre die Einführung von parallelen Registern sowie eine teilweise Verdeutschung von Fachtermini von Bedeutung. Bezüglich des Status könnten sich im Zuge des drastischen Rückgangs des Deutschen auf der internationalen Ebene folgende Maßnahmen als wirksam erweisen: das Deutsche könnte auf Konferenzen zumindest im deutschsprachigen Raum als erste Konferenzsprache fungieren; die identitätsstörende Vorrangstellung des Englischen in Deutschland könnte aufgrund ihrer negativen Auswirkung auf den Status des Deutschen im In- und Ausland wenigstens in Frage gestellt werden; in Russland sei Deutsch als eine gleichberechtigte Fremdsprache neben Englisch, Französisch u. a. anzusehen; statt Geld in die Filialen des Goethe-Instituts fließen zu lassen, müsste man einzelne Personen fördern, die vor Ort aktiv sind (z.B. kenne ich einen Kollegen, der über 9 Jahre lang vergeblich versucht, an Übersetzungsseminaren in Moskau teilzunehmen, dabei ist er kein schlechter Übersetzer; ich selbst arbeite seit über 20 Jahren an einem einzigartigen russisch-deutschen phraseo-parömiologischen Wörterbuch, das ein Desiderat in der russischen Germanistik ist, aber jede Unterstützung bleibt aus); ein deutschsprachiger Rechtschreibwettbewerb, eine aussichtsreiche Idee einer Kollegin aus Tomsk, könnte russlandweit regelmäßig veranstaltet werden.

\section{Auf der Suche nach einer möglichen Lösung}

Um die Frage, wie dem Statusschwund des Deutschen in Russland effektiv entgegenzuwirken sei, zu beantworten, ist ein Verständnis dessen nö- 
tig, wozu die Fremdsprachen überhaupt gelernt werden. Allen Vorstellungen zuwider werden nicht die am meisten verbreiteten Sprachen gelernt, sondern lediglich diejenigen, die im Ausland bzw. auf ein konkretes Land am meisten Einfluss ausüben, der gemeinhin als Prestige bezeichnet wird. Das letztere kann nach einer gültigen Formel berechnet werden [6: 25], jedoch das Entscheidende am Einfluss einer Fremdsprache ist, dass er gesteuert und somit zum Spielball der Interessen von Politikern unterschiedlicher Couleur werden kann. „Es zählt zum größten Manko der Sprachenpolitik, dass sie nicht von Linguisten, sondern von Politikern gemacht wird“", beklagt M. Rannutt [8: 88].

In dieser Hinsicht erweist sich die russlandkritische Rhetorik sämtlicher deutschen Staatsmedien als kontraproduktiv für die deutsche Sprachpolitik in Russland. Statt Anglizismen und allem Amerikanischen Kampf anzusagen, erklären viele proamerikanische Demokraten in der heutigen BRD einen der ältesten deutschen Verbündeten auf dem Kontinent zu einem der größten Risiken für Deutschlands Sicherheit: „Russland ist kein Partner mehr, sondern ein Rivale“ [1]. Glücklicherweise wurde die komplett aus Washington gesteuerte Weißbuchdebatte, die in Russland eine ungeheure Empörungswelle auslöste, mit den versöhnlichen Worten des deutschen Botschafters in Moskau Rüdiger Freiherr von Fritsch vorübergehend beendet: „Wir arbeiten hart für gute Beziehungen“ [10]. Auch wenn der größte Rückschlag für das Prestige der deutschen Sprache dank einem weisen Politiker abgewendet sei, könnte man sich fragen, ob jemand in Russland nach solchen Meldungen noch freiwillig Deutsch lernen würde. Muss man erwarten, dass nach alledem Deutsch bei Russen beliebt sein wird? In einer Situation, wo die gesamte offizielle deutsche Presse vor Russophobie strotzt, muss die Frage im Sinne von G. Grass „was gesagt werden muss“ lieber als „was nicht gesagt werden muss" formuliert werden, um den deutsch-russischen Beziehungen nicht noch stärker zu schaden, die ohnehin durch völlig sinnentleerte Sanktionsmaßnahmen belastet sind.

Wie bereits erwähnt, resultiert die prekäre Lage des Deutschen in Russland aus der Amerikanisierung der deutschen Sprache und Kultur. Dadurch wurde Deutsch leider zum Bestandteil der englischen Sprachpolitik, und solange kein Umdenken bei den deutschen Politikern in Sicht ist, werden die Aussichten fürs Deutsche in Russland immer finsterer. Als Zweitfremdsprache wird es sich schwerlich einleben und, um die erste Fremdsprache (wieder) zu werden, muss es dem Englischen zu viele Kommunikationssphären abringen, darunter Sport, Mode, Freizeit / Lifestyle, Erholung, Wissenschaft, Politik, Finanzen / Bankwesen usw. Die Neologismen, egal in welcher Sprache, werden in diesen Sphären längst aus englischem Morphemmaterial produziert (vgl. [2: 195]) - ein Monopol der englischen Sprache, das nur mit dem des Lateins zu vergleichen wäre.

Die klassische Frage „qui bono?“ (,wem nützt das?") bringt es auf den Punkt: die einzigen Nutznießer der Verbreitung des Deutschen im Aus- 
land sind die Deutschen selbst. Entweder hat man also kein Interesse am Fortbestehen der Deutschtradition im größten Land der Erde oder eben keine Interesse an der eigenen Sprache. Tertium non datur. Dies besagt, dass nur die Deutschen gegenüber vielen Russlandgermanisten Verantwortung tragen, die ihren Beruf unter dem Einfluss der deutschen Sprachenpolitik im Ausland erlernt haben und nun sich im Stich gelassen fühlen. Bei gleichbleibenden sprach(en)politischen Rahmenbedingungen für die deutsche Sprache sind auch solche nennenswerte Initiativen wie Deutsch 3.0 des GoetheInstituts dem Untergang geweiht, obwohl sie sehr engagiert und ambitioniert vorangetrieben wird.

Um wirklich Attraktivität des Deutschen zu steigern, muss auch seitens der DAAD-Lektoren mehr geleistet werden als bloß mit Themen wie „Kulturwissen“ oder „Fremdsprachkompetenzen“ durch Russland zu reisen und auf diese Weise Geld zu verdienen. Die Sprachenpolitik muss von Profis gestaltet werden und nicht von Laien, die einfach nur arbeitslos in Deutschland sind. Um eigene Sprache kämpfen verlangt Mut und Entschlossenheit. Ein altes deutsches Sprichwort lautet: wer mit dem Teufel essen will, muss einen langen Löffel haben - wer mit Englisch konkurrieren will, muss keine Demokratie spielen.

Wenn man heute Englisch lernen will, steht einem die ganze Welt offen, die Angebote überstürzen sich, einem fehlt an nichts, auch nicht am Umgang mit Muttersprachlern, obwohl die Angelsachsen in der Verbreitung des Englischen eher ein Geschäft sehen. Möchte man aber die deutsche Sprache lernen, schlägt einem entweder der enttäuschende Anglizismus "Geoblocking“" in den Mediatheken bei ARD und ZDF oder ein unerschwinglicher Preis auf Euro-Basis entgegen und das bei aller demotivierenden Komplexität der deutschen Grammatik. Welche Filme und wo kann man auf Deutsch schauen außer den Billigprodukten von völlig unauffälligen Kinofestivals, die niemand freiwillig schauen würde? Wenn man sich über den Sport in Deutschland oder olympische Spiele informieren möchte, bekommt man auf seinem Bildschirm nur den abweisenden Satz ,die Inhalte sind in Ihrem Land nicht abrufbar" zu lesen. Einen großen Ausgleich schaffen diesbezüglich lobenswerte Sprachlernangebote der Deutschen Welle, jedoch nicht die Art der Berichterstattung.

Die gesamte Tätigkeit der zeitgenössischen Sprach(en)politiksubjekte steht trotz einer breitgefächerten Palette der Angebote in vielem der Wirksamkeit des Herder-Instituts zu den DDR-Zeiten nach, obwohl es nie zu solchen gehörte. Der Grund bleibt nach wie vor die Amerikanisierung, die trotz aller Kritik an der DDR in den Staaten des Ostblocks keine Chancen hatte.

Heute steht fest: Deutsch scheint - zumindest langfristig - den Kampf um den Status einer künftigen Erstfremdsprache in Russland verloren zu haben. Ein Weiterkämpfen gegen das aggressiv und vollkommen rücksichtslos verbreitete Englisch mit politisch korrekten, demokratischen Mitteln ist nicht 
länger möglich. Es ist nicht die Aufgabe dieses Beitrags, festzustellen, ob die Entwicklung von zentristischen Sprach(en)politikmodellen und eine demokratische Gesellschaft kompatibel wären. Es sei nur darauf hingewiesen, dass eine Sprachenpolitik als Folge der Staats- und Gesellschaftspolitik zwangsläufig in sprach(en)politischen Konzepten existiert, auch wenn dies vielen Sprachnutzern nicht immer bewusst ist. Aus der Sprachenaußenperspektive betrachtet erscheint die heutige amerikazentrische „Demokratie“ in der BRD als ein gewaltiges Hindernis für die Verbreitung der deutschen Sprache und Kultur europaweit.

\section{Literaturverzeichnis}

1. Die Welt. Das sind die größten Risiken für Deutschlands Sicherheit // Die Welt. (2016). URL: http://www.welt.de/politik/deutschland/article155952158/Das-sind-die-groesstenRisiken-fuer-Deutschlands-Sicherheit.html [eingesehen am 20. August 2016].

2. Herberg, D.: Neologismen in der deutschen Gegenwartssprache. Probleme ihrer Erfassung und Beschreibung // DaF, Nr. 4. pp. 195-201. (2002). (In German)

3. Klein, S.: Dümmer auf Englisch. (2007). URL: http://www.faz.net/aktuell/feuilleton/geisteswissenschaften/ wissenschaftssprache-duemmer-auf-englisch-1461421.html [eingesehen am 18. August 2016].

4. Klokov, W.T.: Sprachenpolitik in der afrikanischen Frankophonie. Saratov: Universitätsverlag. (1992). (In Russian)

5. Kobenko, Yu.V.: Exo-endoglossic (Centric) Model of German Language Policy // Journal of Siberian Federal University. Humanities \& Social Sciences. 7. pp. 1015-1024. (2014). (In Russian)

6. Kobenko, Yu.V.: Foreign Languages in Russian Education and Science // Procedia - Social and Behavorial Sciences: Proceedings of XV International Conference „Linguistic and Cultural Studies: Traditions and Innovations“, LKTI 2015, 9-11 November 2015, Tomsk, Russia. Vol. 206. pp. 24-29. (2015). (In Russian)

7. Mattheier, K.J.: Allgemeine Aspekte einer Theorie des Sprachwandels // Sprachgeschichte. Hrsg. v. W. Besch u. O. Reichmann. 1. Hb. Berlin, NY: Walter de Gruyter. pp. 720-730. (2003). (In German)

8. Rannut, M.: Ein Lehrwerk in Sprachenpolitik. Tallinn: Atlex. (2004). (In German)

9. Semenjuk, N.N.: Herausbildung von Literaturnormen und Typen von Kodifizierungsprozessen // Sprachnorm: Typologie von Normalisierungsprozessen. Moskau: Institut für Sprachwissenschaften der Russischen Akademie der Wissenschaften. pp. 23-44. (1996). (In Russian)

10. Sputnik (2016). Deutscher Botschafter in Moskau: „Wir arbeiten hart für gute Beziehungen“ // Sputnik Deutschland. URL: http:// www.de.sputniknews.com/politik/ 20160607/310435000/deutscher-botschafter-fuer-gute-beziehungen.html [eingesehen am 20. Juli 2016].

11. VestiFM. Zwei Fremdsprachen in der Schule: Die Meinungen von Eltern und Experten gingen auseinander. (2015). URL: http://radiovesti.ru/article/show/article_id/176613 [eingesehen am 02. Mai 2016].

12. Viereck, W.: Das Deutsche im Sprachkontakt: Britisches Englisch und Amerikanisches Englisch-Deutsch [Text] // Sprachgeschichte. 1. Bd. Berlin, NY: de Gruyter. pp. 938-948. (1984). (In German) 
CRITICAL REFLEXIONS ON THE CONTEMPORARY GERMAN LANGUAGE POLICY IN RUSSIA

Kobenko Ju.V., National Research Tomsk State University (Tomsk, Russian Federation). Email: serpentis@list.ru

Abstract. The article discusses prerequisites for a balanced German language policy in Russia in the focus of the present language situation in Germany. The focus of the discussion is the antinomy "Exo- vs. Endoglossia" as well as the relevant conceptual apparatus as a synthesis of those of language contact research and sociolinguistics. The main focus here is on balancing language preferences, which should be respected in German language policy with regard to Russia. An essential part of the article is dedicated to the criticism of the existing language policy concept, which is characterized by an overly democratic view of the language-political variables. The analysis results of the current language situation in Russia can be expected in view of a drastic decline in status of the German a stronger language-critical engagement of the public and Russian Germanists and an inevitable revision of the contemporary politics of contemporary politics.

Keywords: German language policy, language criticism, Americanization, language preferences, language policy models

Information about the author:

Juri Kobenko - Doctor in Philology, Professor, Department of German, Faculty of Foreign Languages, National Research Tomsk State University (Tomsk, Russia). E-mail: serpentis@list.ru 